\title{
Double Star Astrometry with the Carlsberg Automatic Meridian Circle
}

\author{
ROBERT W. ARGYLE \\ Royal Greenwich Observatory, Madingley Road, Cambridge, CB3 0EZ, \\ United Kingdom
}

\section{INTRODUCTION}

The Carlsberg Automatic Meridian Circle (CAMC) has been operating on the island of La Palma since 1984. Since that time it has made more than 500,000 observations of stars and minor planets. Observation are made by accumulating the output from a series of scans by a pair of $4^{\prime \prime}$ wide slits which oscillate back and forwards across the star as it traverses the meridian (Helmer et al. 1991). The error of a single determination of a star position is $0^{\prime \prime} .14$ in RA and Dec and 0.05 in $\mathrm{V}$ magnitude. For 20 observations, the errors of a mean position are reduced to about $0^{\prime \prime} .05$.

\section{RADIO STARS}

An important aspect of the work of the CAMC is the linking of the optical and radio reference frames through the observation of radio stars. Measurements have been carried out for several years and some results are given by Morrison et al. (1990). Their brightness makes them ideal objects for measurement by small telescopes but care must be taken in selecting the stars to be observed. Some of the radio stars are double and the presence of a companion affects the measured position. Some such as $\sigma \mathrm{CrB}$ (ADS 9979) can be clearly resolved, but it is by no means clear how many of the several hundred known radio stars are double with separations and magnitude differences which could significantly affect the observed position of the star. Until now, no attempt has been made to extract positional information from observations of double stars which can be resolved by the CAMC (aperture $18 \mathrm{~cm}$ ).

When assessing the effect of known companions on observations with the CAMC the critical distance is the separation of the photocentre from the primary component which depends both on the separation and the magnitude difference of the pair. In practice, pairs with $\Delta m \geq 4$ are treated as single, as are equally bright pairs with separations of $\leq 0^{\prime \prime} 1$.

A survey of radio stars with a speckle interferometer on a large telescope would be most useful in identifying which are unsuitable for optical astrometry.

\section{OBSERVATIONS OF DOUBLE STARS}

In this paper we carry out an assessment of the possibility of observing absolute positions of the components of double stars from CAMC observations. The software currently used to measure the position of the star involves numerical integration of the signal from several scans of the slits across the star. Calcula- 


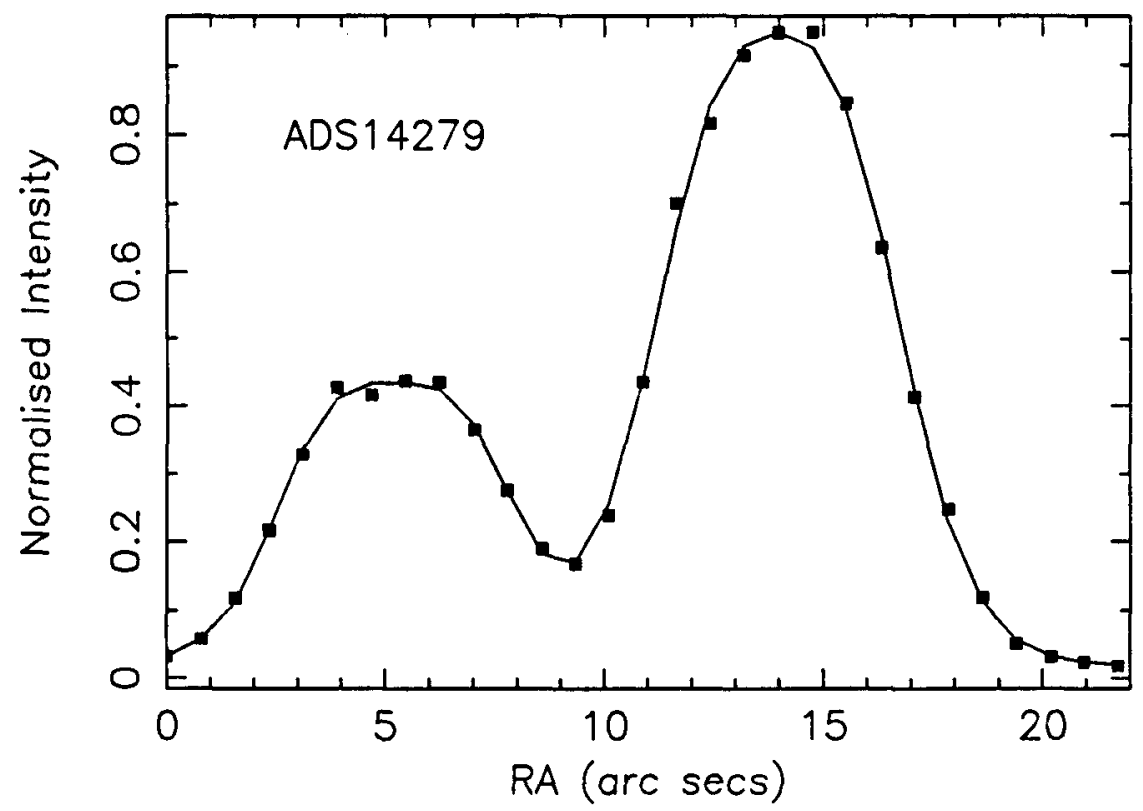

FIGURE 1. CAMC slit scan of ADS 14279.

tion of the third and fourth moments reveal asymmetry or flatness and profiles showing these are rejected from the night's observations on the assumption that they are double. As a result, several hundred observations a year are lost. The CAMC working catalogue contained no information about double star systems apart from a cross-reference to the IDS, so a file of over 21,000 double star components which appear on the CAMC programme using the WDS catalogue (Worley \& Douglass 1984) was created. This includes all pairs which have both components brighter than 14.5 and north of declination $-40^{\circ}$. The file contains the position angle, separation, and difference in magnitude between star $\mathrm{A}$ and $B$ (and vice versa in cases where the fainter of the two stars in a pair is being observed). In systems where there is relative motion, linear extrapolation is used to update the relative coordinates to the current epoch.

The observation of double stars will be approached in two ways. Firstly, for pairs which are $\sim 15$ " apart it is possible to observe both separately by moving the telescope in altitude and adjusting the slit position so as to mask the other component.

Secondly, for double stars closer than 15", a Franz profile (Franz 1973) can be used to deconvolve the blended profiles into two individual profiles for which accurate positions and magnitude differences can be determined. Devaney (1992) has shown that for photometry the Franz function is better than either a Gaussian (which underestimates the wings of the image) or a Lorentzian (which overestimates the wings). In addition, for pairs which are outside the isoplanatic patch, the Franz function has the additional advantage of allowing the profiles of each star to be modelled independently.

The slits are angled at $90^{\circ}$ to each other and $45^{\circ}$ to the horizontal so at least 
one them is well placed to observe both components. Profile fitting to both slits yields measurements of the difference in right ascension and declination and hence position angle $(\theta)$ and separation $(\rho)$ can be calculated.

To ascertain how accurately the separation of double stars can be measured, eight nightly observations were made of the pair ADS 14279 (mean epoch 1990.65) using the CAMC. A typical single slit profile is shown in Figure 1. Linear interpolation of photographic astrometry for this pair from 1948 to 1976 was used to yield a comparison separation for the epoch of observation. The differences between the CAMC measurement of $\theta$ and $\rho$ and those of the photographic position were $-0^{\circ} .6$ and $+0^{\prime \prime} .15$. A contemporaneous micrometer measurement by the author (Argyle 1992) produces residuals of $0^{\circ} .0$ and $-0^{\prime \prime} .08$ from the CAMC values. The relative brightnesses of the two stars can also be obtained. By integrating each of the two CAMC profiles the measured $\Delta m$ is $0.92 \pm 0.02$ mags, compared to a value of $0.94 \pm 0.03$ quoted by Wierzbinski (1969). The centre of each profile can be related to the FK5 system by using the telescope constants which are kept after the reduction of each night's observing.

\section{SUMMARY}

The use of Franz profiles promises to allow measurement of the absolute positions for both stars in binary systems provided they are sufficiently well separated. The circle-reading photomultipliers are being replaced by CCD cameras which will increase both the speed of operation and the accuracy of setting and observing. This will allow shorter slit lengths to be used and hence closer pairs $\left(\sim 6^{\prime \prime}\right)$ could be observed separately.

\section{ACKNOWLEDGEMENTS}

The CAMC is operated jointly by the Copenhagen University Observatory, the Royal Greenwich Observatory and the Real Instituto y Observatorio de la Armada en San Fernando.

\section{REFERENCES}

Argyle, R.W. 1992, Webb Society Double Star Section Circulars, 5, in press

Devaney, N. 1992, $A \& A$, in press

Franz, O.G. 1973, JRASC, 67, 81

Helmer, L., Fabricius, C., \& Morrison, L.V. 1991, Exper. Astron., 2, 85

Morrison, L.V., Argyle, R.W., Requième, Y., \& Mazurier, J.M. 1990, A\&A, 236, 256

Wierzbinski, S. 1969, Contrib. Wrocław Astronomical Observatory, 16

Worley, C.E. \& Douglass, G.G. 1984, The Washington Double Star Catalogue, 1984.0 